\title{
Inhibitory effects of canagliflozin on pancreatic cancer are mediated via the downregulation of glucose transporter-1 and lactate dehydrogenase $\mathrm{A}$
}

\author{
DUIYUE XU ${ }^{1 *}$, YIRAN ZHOU $^{2 *}$, XIN XIE $^{1}$, LIANGYUAN HE ${ }^{1}$, JIALU DING $^{1}$, \\ SHUYANG PANG ${ }^{1}$, BAIYONG SHEN ${ }^{2}$ and CHANGLIN ZHOU ${ }^{1}$ \\ ${ }^{1}$ School of Life Science and Technology, China Pharmaceutical University, Nanjing, Jiangsu 211198; \\ ${ }^{2}$ Department of General Surgery, Rui Jin Hospital, Research Institute of Pancreatic Diseases, \\ School of Medicine, Shanghai JiaoTong University, Shanghai 200025, P.R. China
}

Received February 6, 2020; Accepted August 25, 2020

DOI: 10.3892/ijo.2020.5120

\begin{abstract}
Pancreatic cancer is one of the most lethal solid malignancies, with a poor prognosis and a high mortality rate. Pancreatic cancer cells exhibit enhanced glycolysis to maintain their rapid growth. Canagliflozin (CANA) is a sodium-glucose co-transporter 2 inhibitor used for the clinical treatment of diabetes. Recent studies have demonstrated the potential ability of CANA to suppress hepatocellular carcinoma, whereas its therapeutic effects on and mechanisms in pancreatic cancer have rarely been reported. In the present study, the antitumor effects of CANA on pancreatic cancer were investigated. The data obtained indicated that pancreatic cancer growth was effectively suppressed by CANA in a dose-dependent manner, with peak inhibition rates of 54.3 and $57.6 \%$ in cultured Capan-1 and PANC-1 cells respectively. The tumor inhibitory rate reached $45.2 \%$ in nude mice with PANC-1-derived tumors, suggesting its effective antitumor activity against pancreatic cancer in vitro and/or in vivo. In addition, the combined treatment of Capan-1 and PANC-1 cells with gemcitabine and CANA exhibited a greater efficacy compared with that of treatment with gemcitabine alone. Moreover, glucose uptake and lactate production were
\end{abstract}

Correspondence to: Professor Changlin Zhou, School of Life Science and Technology, China Pharmaceutical University, 639 Longmian Road, Nanjing, Jiangsu 211198, P.R. China

E-mail: cl_zhou@cpu.edu.cn

Professor Baiyong Shen, Department of General Surgery, Rui Jin Hospital, Research Institute of Pancreatic Diseases, School of Medicine, Shanghai JiaoTong University, Shanghai 200025, P.R. China

E-mail: shenby@shsmu.edu.cn

*Contributed equally

Key words: pancreatic cancer, canagliflozin, glycolysis, glucose transporter-1, lactate dehydrogenase A decreased, and the mRNA levels of the glycolysis-associated genes, including glucose transporter-1 and lactate dehydrogenase A were decreased, indicating the inhibitory effects caused by the combination treatment on the metabolism of glucose in pancreatic cancer cells. Furthermore, CANA induced apoptosis, notably early apoptosis, and decreased the protein levels of PI3K, p-AKT, p-mTOR and HIF-1 $\alpha$, which indicated that the PI3K/AKT/mTOR signaling pathway was involved in the glycolytic process. These results demonstrated that pancreatic cancer growth was effectively inhibited by CANA via the suppression of glycolysis. This was mediated primarily by the $\mathrm{PI} 3 \mathrm{~K} / \mathrm{AKT} / \mathrm{mTOR}$ signaling pathway, revealing the underlying role and potential of this pathway for the clinical treatment of pancreatic cancer. Novel applications for the existing drug CANA can be explored, which could reduce the cost and time required for drug development in the field of drug discovery.

\section{Introduction}

Pancreatic cancer is one of the leading causes of cancer-related mortality, with a 5-year survival rate of $8 \%$. This disease is expected to surpass breast malignancy as the third leading cause of cancer-related mortality in the USA $(1,2)$. Routine examinations of the pancreas are not readily performed and consequently, the low survival rate noted in patients with pancreatic cancer is largely due to the advanced stage of disease at diagnosis (3). To date, gemcitabine has been used as the standard treatment for pancreatic cancer, extending patient survival by only a few months with several adverse reactions, such as atrophy of hematopoietic organs and immune suppression (4). Therefore, the development of novel effective strategies to control pancreatic cancer is urgently required.

Cancer cells always reprogram their metabolism and enhance glycolysis to meet the constantly growing requirement for intermediates, even in the presence of abundant oxygen, which is known as the Warburg effect $(5,6)$. Recently, studies have indicated that the activation of the PI3K/AKT signaling pathway can promote pancreatic cancer proliferation and that the expression of several genes involved in glycolysis can be upregulated by oncogenes, such as PI3K/Akt, NF- $\mathrm{kB}$ and 
c-myc $(7,8)$. Enzymes, such as glucose transporter-1 (GLUT-1), lactate dehydrogenase A (LDHA) and monocarboxylic acid transporter- 4 , which are highly expressed in tumors and are associated with a poor prognosis, are involved in the glycolytic process (9). There is also accumulating evidence to suggest that the inhibition of key enzymes, such as GLUT-1 and LDHA, can effectively delay tumor progression $(10,11)$.

Based on known pharmacokinetic and safety profiles, the existing clinical drugs can be further screened to identify potential new indications that can significantly reduce the cost and time of drug exploration. This method is considered one of the most rapid and effective strategies in the field of drug discovery. Certain agents can alter glucose metabolism or inhibit the expression of glucose transporters to suppress tumor growth (12). Canagliflozin (CANA) is an important oral medication used for diabetes that acts by inhibiting the activity of sodium-glucose cotransporter 2 (13). Recent studies have demonstrated that CANA may suppress liver cancer progression, whereas the effects of CANA against pancreatic cancer have not been fully investigated $(14,15)$.

In the present study, pancreatic cancer cells and a PANC-1-derived xenograft tumor model were used to assess the antitumor activity of CANA. The results suggested that CANA was capable of suppressing pancreatic cancer growth through the inhibition of glycolysis mediated primarily via the $\mathrm{PI} 3 \mathrm{~K} / \mathrm{AKT} / \mathrm{mTOR}$ signaling pathway. These findings indicate its potential use in the clinical treatment of pancreatic cancer.

\section{Materials and methods}

Cell culture and reagents. The two human pancreatic cancer cell lines, Capan-1 and PANC-1 (American Type Culture Collection), were cultured in Dulbecco's modified Eagle's medium with high glucose (Gibco; Thermo Fisher Scientific, Inc.) supplemented with $10 \%$ FBS, $100 \mathrm{U} / \mathrm{ml}$ penicillin and $100 \mu \mathrm{g} / \mathrm{ml}$ streptomycin (Beyotime Institute of Biotechnology) in a humidified incubator containing $5 \% \mathrm{CO}_{2}$ at $37^{\circ} \mathrm{C}$. CANA (Meilun Biotechnology), gemcitabine (Luoxin Biotechnology), LY294002 (MedChem Express) were dissolved in DMSO or physiological saline for use in the in vitro experiments.

Cell viability assay. The viability of the Capan-1 and PANC-1 cells was assessed by MTT assay (Sigma-Aldrich; Merck KGaA). Briefly, the cells were seeded in a 96-well plate at a density of 5,000 cells/well, incubated for $24 \mathrm{~h}$ and treated with $20,40,60$ and $80 \mu \mathrm{M}$ CANA, or $0.5 \mu \mathrm{M}$ gemcitabine for $48 \mathrm{~h}$. The number of viable cells was quantified by measuring the OD at $490 \mathrm{~nm}$ with a microplate reader (AC100; Meigu Molecular Instruments Co., Ltd.) and the results were expressed as a percentage (\%) of the control. The IC50 values were calculated using GraphPad Prism 4.0 software (GraphPad Software).

Colony formation assay. The Capan-1 and PANC-1 cells were seeded in 6-well plates at a density of 500 cells/well in $2 \mathrm{ml}$ culture medium. Following $72 \mathrm{~h}$ of incubation $\left(37^{\circ} \mathrm{C}\right.$, $5 \% \mathrm{CO}_{2}$ ), the cells were treated with 20,40 and $60 \mu \mathrm{M}$ CANA, or $0.03 \mu \mathrm{M} 22$ gemcitabine, or treated with the combination of $20 \mu \mathrm{M}$ CANA and $0.03 \mu \mathrm{M}$ gemcitabine for 10 days $\left(37^{\circ} \mathrm{C}, 5 \% \mathrm{CO}_{2}\right)$. The cells were then rinsed with $1 \mathrm{X}$
PBS, fixed with chilled at room temperature for $10 \mathrm{~min}$ and stained with $0.5 \%$ crystal violet (Beijing Solarbio Science \& Technology Co., Ltd.) at $25^{\circ} \mathrm{C}$ for $40 \mathrm{~min}$. Cells morphology was observed and photographed under a light microscope (Nicon Corporation). The experiments were independently performed in triplicate.

Flow cytometric analysis. The ontrol and treated cells were stained with an Annexin V/propidium iodide staining kit (Vazyme) to assess cell death according to the manufacturer's instructions. The stained cells were captured by flow cytometry (BD Biosciences) and 10,000 events were collected. All assays were repeated at least 3 times.

Glucose uptake and lactate release assay. The DMEM culture medium contains high glucose, which is taken up by cells to provide energy and produce lactate. The supernatants from the control and treated cell cultures (40 and $60 \mu \mathrm{M}$ CANA) were collected at 6,12 and $24 \mathrm{~h}$. The glucose uptake rate was measured using a glucose assay kit (Jiancheng) according to the manufacturer's instructions. Lactate release was determined using a lactate assay kit (Beijing Solarbio Science \& Technology Co., Ltd.).

In vivo antitumor activity assay. The PANC-1 cells (100 $\mu 1$, $6 \times 10^{6}$ cells $/ \mathrm{ml}$ ) were suspended in saline and injected into the underarms of 4-week-old male nude mice (weigh 18-20 g, $\mathrm{n}=25$ in total) (Balb/c, Yangzhou University, Jiangsu, China). The mice were kept in an environment with a temperature of $22 \pm 1^{\circ} \mathrm{C} / 40 \%$ relative humidity and light exposure $12 \mathrm{~h} /$ day. They were provided with sufficient food and water. All animal experiments were performed according to the protocols approved by the Ethics Committee of China Pharmaceutical University. After 1 week, the xenograft mice were randomly divided into 5 groups according to the tumor volume. Each group included 5 mice, with 25 mice in total. Mice in group 1 received the vehicle (physiological saline, $10 \mathrm{ml} / \mathrm{kg}$ ), those in group 2 were treated with an intraperitoneal injection of gemcitabine at $10 \mathrm{mg} / \mathrm{kg}$ twice a week, and those in groups 3 , 4 and 5 were orally administered CANA at doses of 25, 50 and $100 \mathrm{mg} / \mathrm{kg}$ every day for 5 weeks. Gemcitabine was dissolved in normal saline and CANA was resuspended in CMC-Na solution. All mice involved in the experiment were euthanized by cervical dislocation following the final administration of the drugs and the sizes of the collected tumors were recorded. The tumor tissues were stored in a refrigerator at $-80^{\circ} \mathrm{C}$. For hematoxylin and eosin (H\&E) staining, a standard staining procedure was performed. In brief, tumor tissues were fixed with $10 \%$ formalin at room temperature for $24 \mathrm{~h}, 6-\mu \mathrm{m}$-thick slices were prepared. Subsequently, hematoxylin staining was lasted for $3 \mathrm{~min}$, as well as eosin staining for $3 \mathrm{~min}$ (at room temperature). Finally, the specimens were dried and observed under an inverted microscope (Nikon Corporation).

Reverse transcription-quantitative PCR (RT-qPCR) analysis. Total RNA was extracted from the control and treated cells using TRIzol reagent. The RNA samples were transcribed into cDNA with a Reverse Transcription kit (Vazyme). The qPCR assays were performed with a ChamQ SYBR qPCR Master 
Mix kit (Vazyme) and analyzed on the StepOne ${ }^{\text {TM }}$ Real-Time PCR system (Thermo Fisher Scientific, Inc.) All procedures were conducted according to the manufacturers' protocols with $5 \mu 12$ X ChamQ SYBR qPCR Master Mix, $0.2 \mu 1$ 50X ROX Reference Dye1, $2 \mu 1$ template cDNA (diluted with nuclease-free water), $0.2 \mu \mathrm{l}$ primer $1(10 \mu \mathrm{M}), 0.2 \mu \mathrm{l}$ primer 2

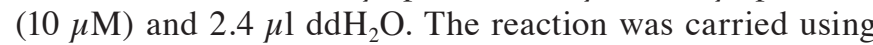
specific thermal cycling conditions, including the 3 following stages: Stage $1,95^{\circ} \mathrm{C}$ for $3 \mathrm{~min}$; stage $2,95^{\circ} \mathrm{C}$ for $10 \mathrm{sec}, 56^{\circ} \mathrm{C}$ for $30 \mathrm{sec}$ and $72^{\circ} \mathrm{C}$ for $30 \mathrm{sec}$; stage $3,95^{\circ} \mathrm{C}$ for $15 \mathrm{sec}, 60^{\circ} \mathrm{C}$ for $60 \mathrm{sec}$ and $95^{\circ} \mathrm{C}$ for $15 \mathrm{sec}$. Each gene primer sequence was designed as follows: GAPDH forward, 5'-AGGTCGGTG TGAACGGATTTG-3' and reverse, 5'-TGTAGACCATGT AGTTGAGGTCA-3'; aldolase A (ALDOA) forward, 5'-ATG CCCTACCAATATCCAGC-3' and reverse, 5'-GACAGCCCA TCCAACCCT-3'; glucose phosphate isomerase (GPI) forward, 5'-CAAGGACCGCTTCAACCACTT-3' and reverse, 5'-CCA GGATGGGTGTGTTTGACC-3'; enolase 2 (ENO2) forward, 5'-CGTTACTTAGGCAAAGGTGTCC-3' and reverse, 5'-CTC CAGCATCAGGTTGTCCAGT-3'; LDHA forward, 5'-ATG GCAACTCTAAAGGATCAGC-3' and reverse, 5'-CCAACC CCAACAACTGTAATCT-3'; GLUT-1 forward, 5'-CGGGCC AAGAGTGTGCTAAA-3' and reverse, 5'-TGACGATACCGG AGCCAATG-3'; and phosphofructokinase-1 (PFK1) forward, 5'-GCCATCAGCCTTTGACAGA-3' and reverse, 5'-CTC CAAAAGTGCCATCACTG-3'. The relative mRNA expression levels were normalized to those of the GAPDH and were determined using the comparative Cq method $\left(2^{-\Delta \Delta \mathrm{Cq}}\right)(16)$.

Western blot analysis. The cells were plated in 6-well plates and collected for protein extraction following treatment with $60 \mu \mathrm{M}$ CANA and the PI3K/AKT/mTOR inhibitor, LY294002 ( $25 \mu \mathrm{M}$, MedChem Express) for $24 \mathrm{~h}$ (17). The cells were lysed in RIPA buffer in the presence of protease inhibitor cocktail. The protein concentration was determined using a BCA assay (Beyotime Institute of Biotechnology). The cell lysates were subjected to SDS-PAGE gels ( $8 \%$ polyacrylamide separation gel and 5\% concentrated gel) and transferred onto the PVDF membrane to separate proteins (50 $\mu \mathrm{g}$ per lane). The following primary antibodies were used: Mouse anti- $\beta$-actin (1:500; cat. no. sc-8432), mouse anti-hypoxia-inducible factor (HIF)-1 $\alpha$ (1:500; cat. no. sc-13515), mouse anti-GLUT-1 (1:500; cat. no. sc-377228), mouse anti-LDHA (1:500; cat. no. sc-137243), rabbit anti-phosphorylated (p-)mammalian target of rapamycin (p-mTOR; 1:1,000; cat. no. sc-293133), rabbit anti-mTOR (1:1,000; cat. no. sc-517464; all from Santa Cruz Biotechnology, Inc.), rabbit anti-PI3K (1:1,000; cat. no. 3011), rabbit anti-p-AKT (1:2,000; cat. no. 4060) and rabbit anti-AKT (1:1,000; cat. no. 4691; all from Cell Signaling Technology, Inc.). The blots were detected using a Bio-Imaging System (Bio-Rad Laboratories, Inc.) and the ImageJ software was used for expression analysis (http://rsbweb.nih.gov/ij/).

Statistical analysis. GraphPad Prism 4.0 software (GraphPad Software) was used for all statistical analyses. The significant differences between 2 groups were examined using the Student's t-test. Statistical comparisons between $\geq 3$ groups were tested using one-way analysis of variance (ANOVA), followed by Tukey's multiple comparison test. The data are presented as the means $\pm \mathrm{SD}$. $\mathrm{P}<0.05$ was considered to indicate a statistically significant difference.

\section{Results}

Pancreatic cancer is effectively suppressed by CANA in vitro. To examine the effects of CANA against pancreatic cancer, the Capan-1 and PANC-1 cells were incubated with CANA for $48 \mathrm{~h}$ and the viability of the cells was measured by MTT assay. The viability of the Capan-1 and PANC-1 cells was decreased by CANA in a dose-dependent manner, with peak inhibition rates of 54.3 and $57.6 \%$ in the cultured Capan-1 and PANC-1 cells, respectively treated with $80 \mu \mathrm{M}$ CANA (Fig. 1A). The $\mathrm{IC}_{50}$ values in the Capan-1 and PANC-1 cells were 77 and $68 \mu \mathrm{M}$, respectively. The inhibitory rate increased from 21.5 and $26.6 \%$ to 29.8 and $36.4 \%$ in the Capan-1 and PANC-1 cells, respectively, following combined treatment with $20 \mu \mathrm{M}$ CANA and gemcitabine compared to treatment with gemcitabine $(0.25 \mu \mathrm{M})$ alone (Fig. 1B). The data indicated that cell proliferation was effectively inhibited by the combination of gemcitabine and CANA compared with single treatment of the cells with either compound. Moreover, the number of cells was decreased and the morphology of the PANC-1 cells was altered from a round to an elongated and shrunken shape following the treatment with $60 \mu \mathrm{M}$ CANA (Fig. 1C). The ability of $60 \mu \mathrm{M}$ CANA treatment to inhibit the colony formation of the cancer cells was comparable to that of the positive control drug (Fig. 1D and E). Combined treatment with $0.03 \mu \mathrm{M}$ gemcitabine and $20 \mu \mathrm{M}$ CANA significantly increased the ability of gemcitabine to inhibit the colony formation of the Capan-1 cells compared to treatment with gemcitabine alone (Fig. $1 \mathrm{~F}$ and $\mathrm{G}$ ). These data suggested that CANA inhibited pancreatic cancer cell growth and colony formation. Moreover, CANA enhanced the ability of gemcitabine to inhibit pancreatic cancer cell proliferation and colony formation.

CANA induces pancreatic cancer cell apoptosis. To further examine whether CANA suppresses cell growth by inducing cell apoptosis, the tumor cells were stained with Annexin V/propidium iodide following drug administration and cell apoptosis was detected by flow cytometry. As shown in Fig. 2, the data in the flow cytometry plots are representative images, while the data in the bar charts represent the overall data. Thus, the data shown in the plots and graphs do not necessarily represent each other. Following treatment with $60 \mu \mathrm{M}$ CANA, the apoptotic rate of the Capan-1 and PANC-1 cells, notably of the early apoptotic cells, was significantly increased, reaching 13.2 and $11.3 \%$, respectively (Fig. 2A and B). However, the percentage of late apoptotic PANC-1 cells exhibited a more obvious effect (compared to the control) following treatment with $60 \mu \mathrm{M}$ CANA than that of the late apoptotic Capan-1 cells (Fig. 2C), suggesting that the PANC-1 cells may be more sensitive to CANA than the Capan-1 cells. Moreover, the apoptotic rate of the Capan-1 and PANC-1 cells increased in a dose-dependent manner, and the apoptotic rate of the $40 \mu \mathrm{M}$ CANA-treated group reached $18.4 \%$ in the Capan-1 cells, while that of the GEM-treated group was only $14.2 \%$ (Fig. 2D). The early apoptotic rate of the Capan-1 cells was significantly increased when 
A

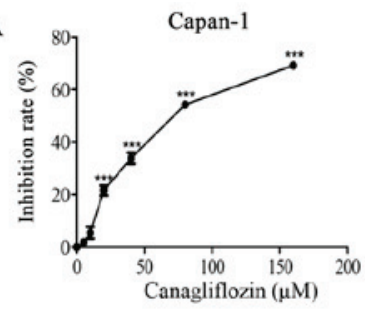

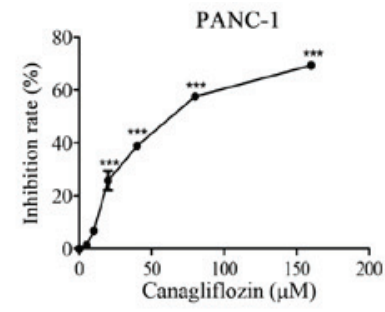

B

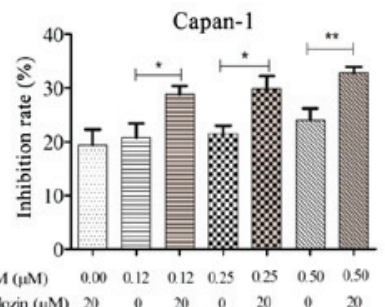

PANC-1

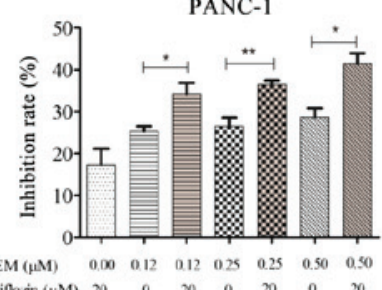

C
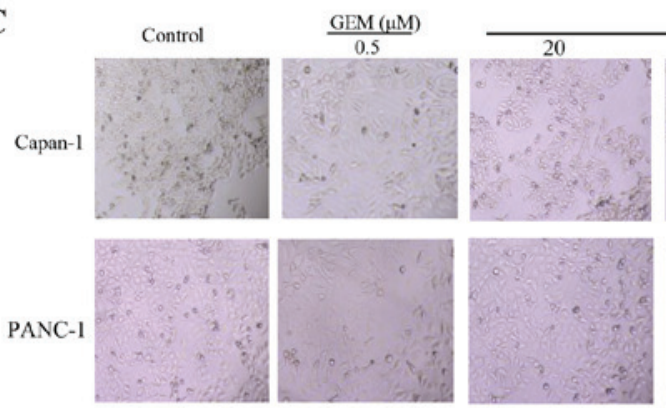

Canagliflozin $(\mu \mathrm{M})$
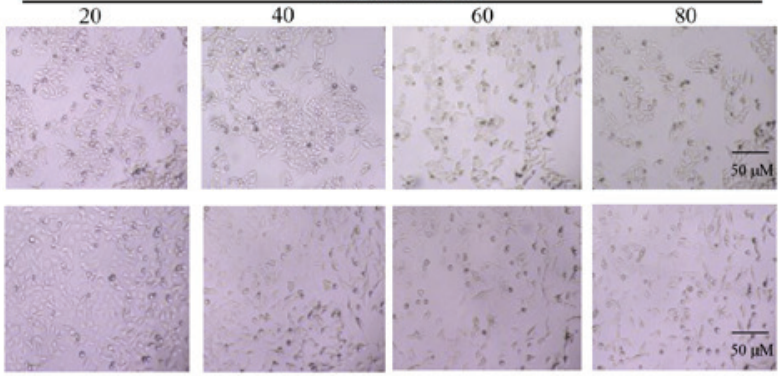

D

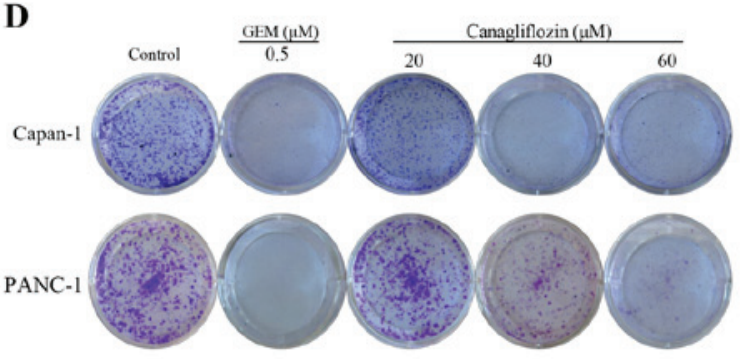

E
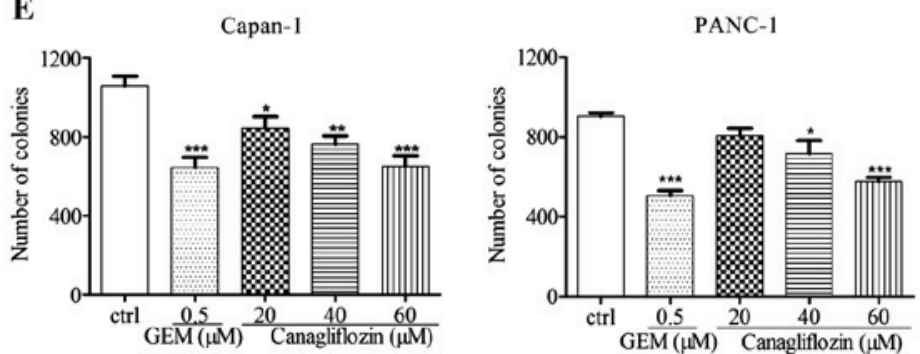

F

G

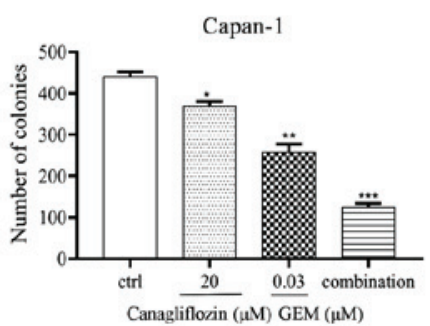

Figure 1. Growth and colony formation are effectively inhibited by CANA in cultured pancreatic cancer cells. (A) Capan-1 and PANC-1 cells were treated with CANA at 48 h, and cell viability was measured by MTT assay. (B) Proliferation of Capan-1 and PANC-1 cells treated with gemcitabine alone or combined with CANA was assessed by MTT assay. (C) The morphological changes of Capan-1 and PANC-1 cells were observed by light microscopy (magnification, x200) at 48 h. (D-G) Colony formation assays were performed to determine the ability of cells to form colonies. ${ }^{*} \mathrm{P}<0.05,{ }^{* *} \mathrm{P}<0.01$ and ${ }^{* * *} \mathrm{P}<0.001$. CANA, canagliflozin.

gemcitabine $(0.5 \mu \mathrm{M})$ was used in combination with CANA $(20 \mu \mathrm{M})$. However, the late apoptotic rate of the PANC-1 cells was markedly improved with this treatment combination (Fig. 2F and G). The total apoptotic rate of the Capan-1 and PANC-1 cells was significantly increased from 11.8 and $12.5 \%$ to 14.9 and $16.3 \%$, respectively when gemcitabine was used in combination with CANA (Fig. 2E and H). These data suggested that CANA inhibited tumor growth by inducing the apoptosis of pancreatic cancer cells. Moreover, CANA enhanced the effects of gemcitabine on the induction of the apoptosis of pancreatic cancer cells.

Rate of glycolysis in pancreatic cancer cells is inhibited by treatment with CANA via the PI3K/AKT/mTOR pathway. The majority of tumor cells exhibit the constitutive upregulation of glycolysis, which is known as the Warburg effect (18).
Tumor cells meet their own energy demands through glycolysis. Since CANA is a metabolism-associated drug, it was hypothesized that it may affect glycolysis in tumor cells. In the present study, the glucose uptake and lactate release rates were examined in the Capan-1 and PANC-1 cells for a certain period of time following treatment with the drugs. It is interesting to note that the glucose uptake rate was reduced in the CANA-treated group (60 $\mu \mathrm{M}$ CANA) of Capan-1 and PANC-1 cells by 4.8 and $4.9 \%$, respectively compared with that of the control group (Fig. 3A). The lactate release rate was reduced by 25.5 and $43.4 \%$ (Fig. 3B). In addition, the mRNA expression levels of glycolysis-associated genes were detected by RT-qPCR at $24 \mathrm{~h}$ following treatment of the Capan-1 and PANC-1 cells with CANA. Specifically, the mRNA expression levels of GLUT-1 and LDHA were markedly decreased (Fig. 3C). CANA was thus capable of 

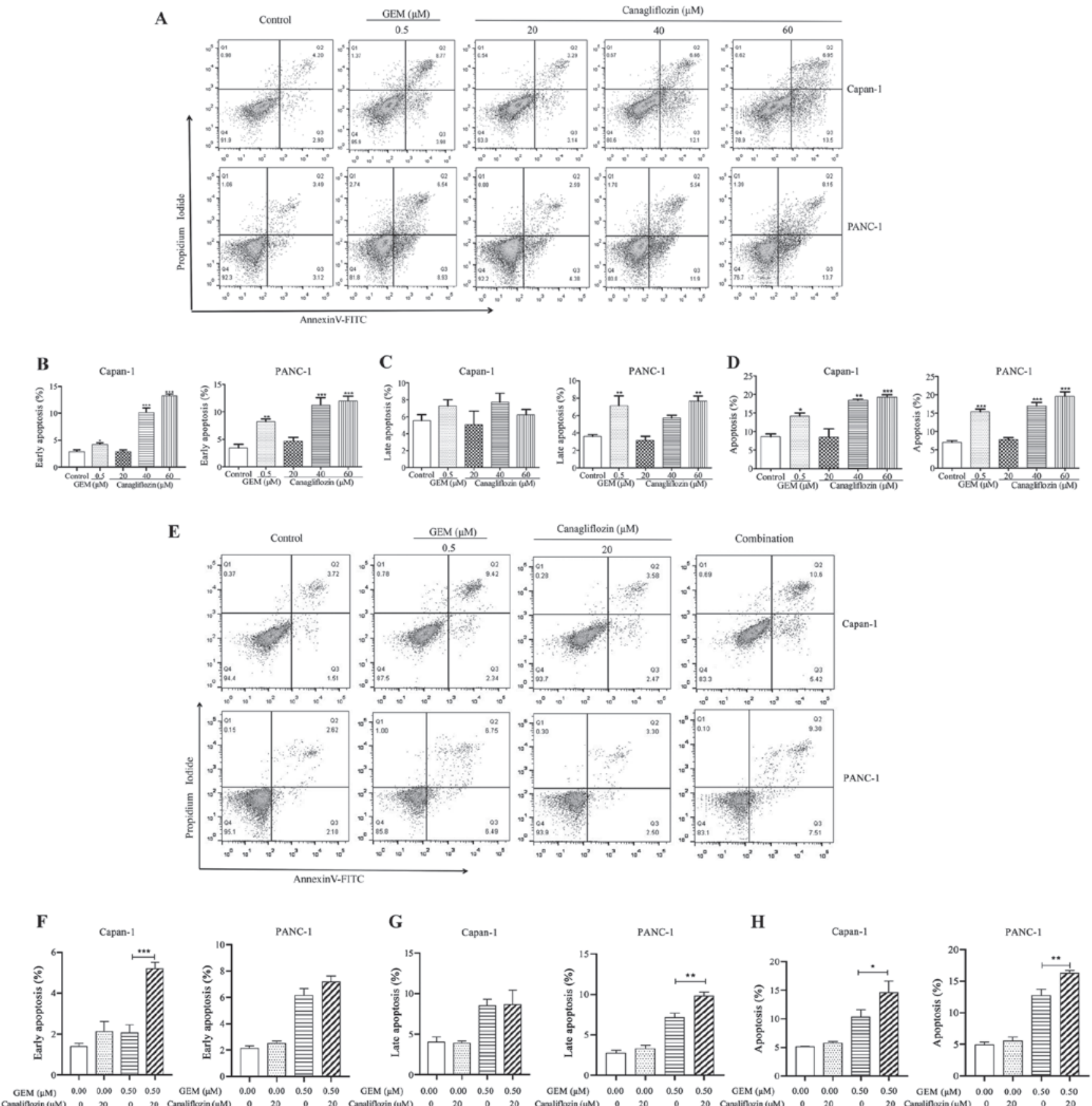

Figure 2. CANA induces apoptosis in pancreatic cancer cells. (A and E) Capan-1 and PANC-1 cells were treated with CANA alone or in combination with gemcitabine. The apoptotic rate of Capan-1 and PANC-1 cells was measured by an Annexin V (AV) and the propidium iodide (PI) apoptosis detection kit. (B-D and F-H) The percentages of apoptosis are shown. The data in the flow cytometry plots are representative images, while the data in the bar charts represent the overall data. Thus, the data in the plots and graphs do not necessarily represent each other. ${ }^{*} \mathrm{P}<0.05,{ }^{* *} \mathrm{P}<0.01$ and ${ }^{* * * *} \mathrm{P}<0.001$. CANA, canagliflozin.

inhibiting glycolysis by downregulating GLUT-1 and LDHA in pancreatic cancer cells.

The PI3K/AKT/mTOR pathway is involved in the glycolytic process and HIF-1 $\alpha$ is a major transcriptional regulator of the response to hypoxia that can induce several genes encoding glycolytic enzymes (19). Therefore, the protein levels of PI3K, p-AKT, p-mTOR, HIF-1 $\alpha$, GLUT-1 and LDHA were determined by western blot analysis in the control and treatment groups. The data indicated that CANA not only inhibited the PI3K, AKT and mTOR phosphorylation reactions, but it also downregulated HIF-1 $\alpha$, GLUT-1 and LDHA expression. In addition, the Capan-1 and PANC-1 cells were treated with the PI3K/AKT/mTOR inhibitor, LY294002, for $24 \mathrm{~h}$ and the protein expression levels of PI3K, P-AKT, P-mTOR, HIF-1 $\alpha$, GLUT-1 and LDHA were apparently inhibited, as noted following treatment of the cells with CANA (Fig. 3D and E). In addition, effects of the combination of CANA with the PI3K/AKT pathway inhibitor, LY294002, on the apoptosis of the PANC-1 and Capan-1 cells were also investigated. As shown in Fig. 3F-I, the data in the flow cytometry plots are representative images, while the data in the bar charts represent the overall 
A

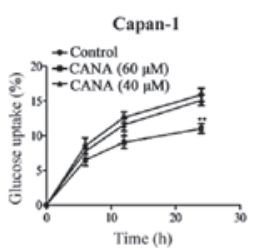

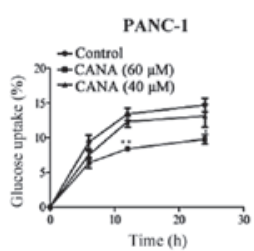

B
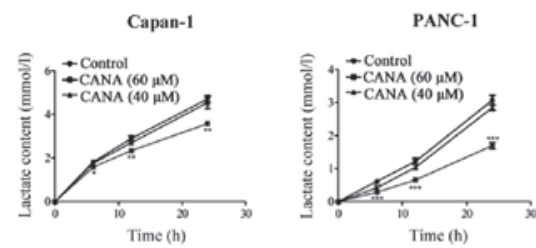

C
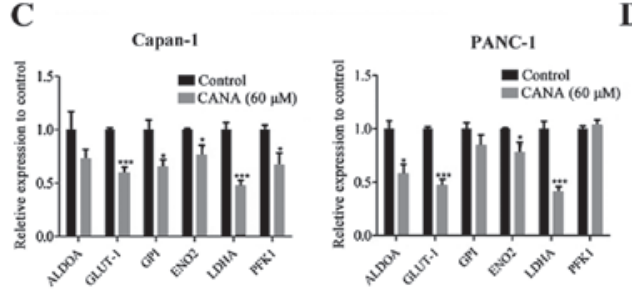

D
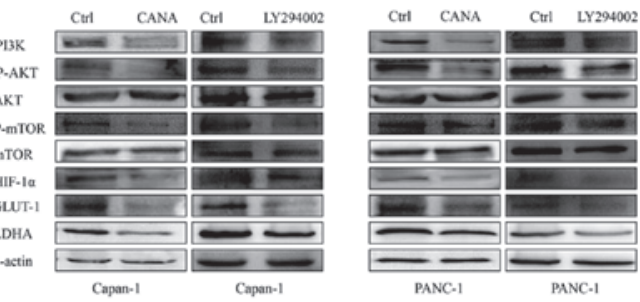

$\mathbf{E}$
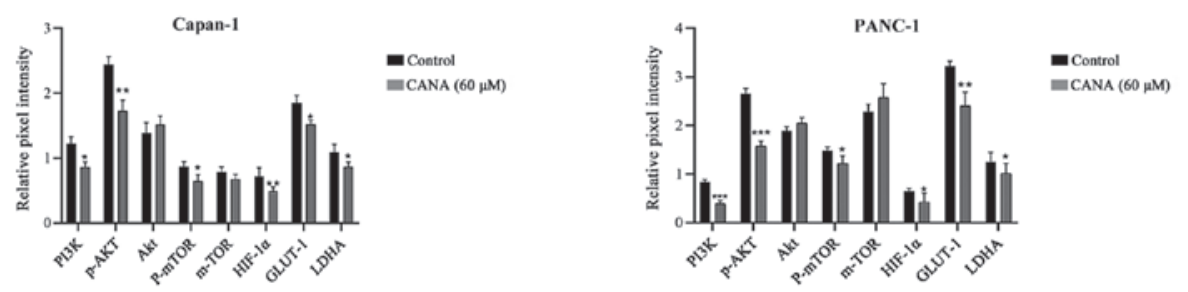

$\mathbf{F}$
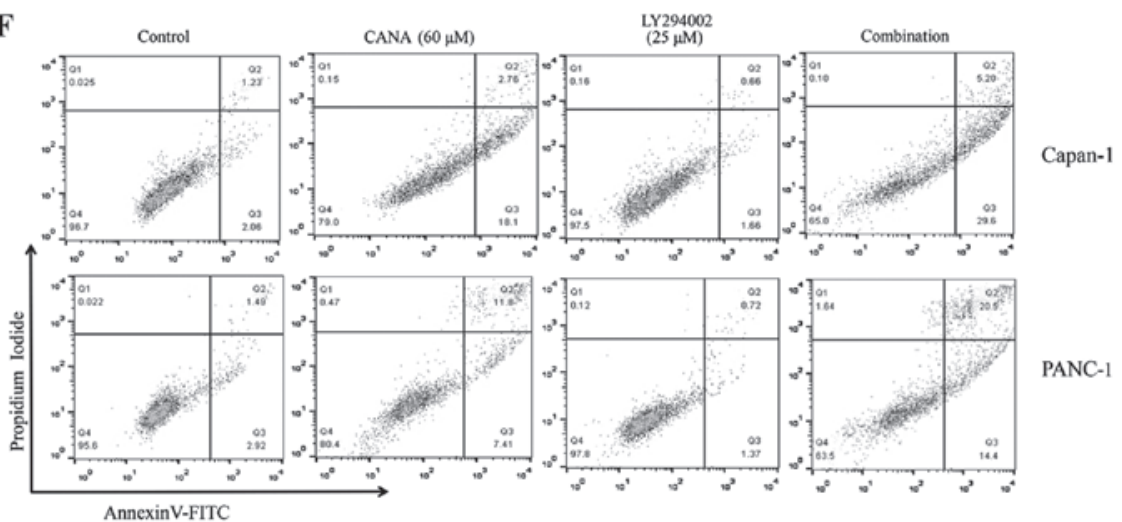

G
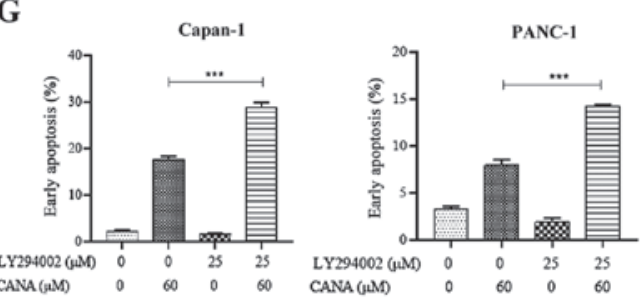

H
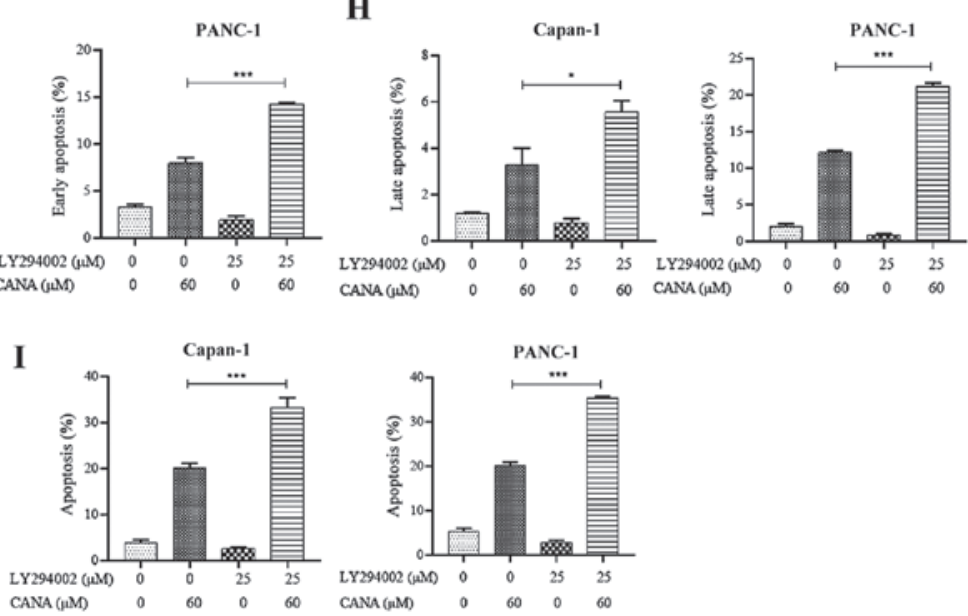

Figure 3. Glucose uptake, lactate release, GLUT-1 and LDHA expression and PI3K/AKT/mTOR signaling were inhibited by CANA in cultured Capan-1 and PANC-1 cells. The concentrations of glucose and lactate in the supernatant were assessed at 6,12 and $24 \mathrm{~h}$. (A) The glucose uptake rate was measured in the control and treated groups of Capan-1 and PANC-1 cells. (B) Lactate release of control and treated cells. (C) Capan-1 and PANC-1 cells were treated with $60 \mu \mathrm{M}$ CANA. Total RNA was extracted and the mRNA expression levels of ALDOA, GLUT-1, GPI, ENO2, LDHA and PFK1 were analyzed by RT-qPCR. (D) The protein levels of PI3K, p-AKT, p-mTOR, HIF-1 $\alpha$, GLUT-1 and LDHA were determined by western blot analysis. (E) The relative expression levels were normalized to the control. (F) Capan-1 and PANC-1 cells were treated with CANA alone or in combination with LY294002. The apoptotic rate of Capan-1 and PANC-1 cells was measured by an Annexin V (AV)/propidium iodide (PI) apoptosis detection kit. (G-I) The percentages of apoptotic cell are quantified The data in the flow cytometry plots are representative images, while the data in the bar charts represent the overall data. Thus, the data in the plots and graphs do not necessarily represent each other. The data are presented as the means $\pm \mathrm{SD}$ of 3 independent experiments. ${ }^{*} \mathrm{P}<0.05,{ }^{* * *} \mathrm{P}<0.01$ and ${ }^{* * * *} \mathrm{P}<0.001$ compared to the control. GLUT-1, glucose transporter-1; LDHA, lactate dehydrogenase A; CANA, canagliflozin; RT-qPCR, reverse transcription-quantitative PCR. 
$\mathbf{A}$

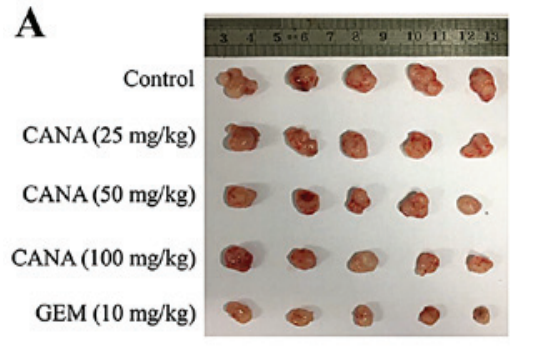

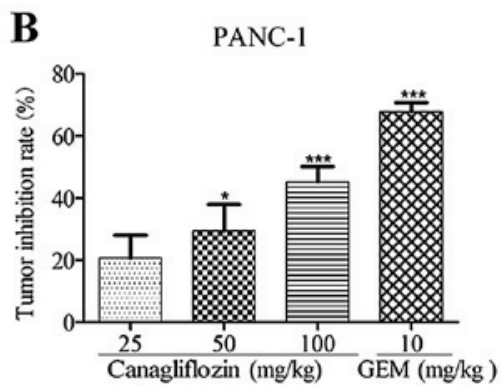

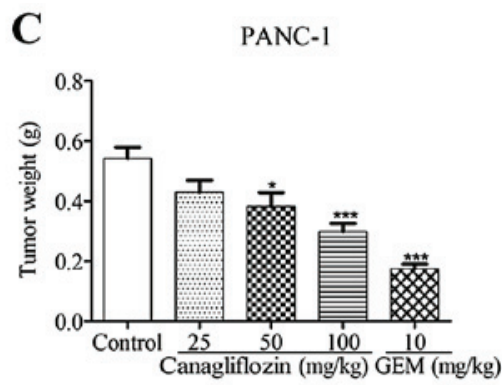

D

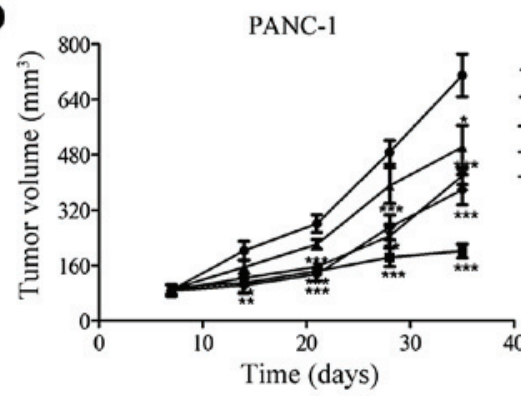

E

- Control - $10 \mathrm{mg} / \mathrm{kg} \mathrm{GEM}$ - $25 \mathrm{mg} / \mathrm{kg}$ CANA + $50 \mathrm{mg} / \mathrm{kg}$ CANA $\rightarrow 50 \mathrm{mg} / \mathrm{kg}$ CANA
$\rightarrow \quad 100 \mathrm{mg} / \mathrm{kg}$ CANA

$\mathbf{F}$
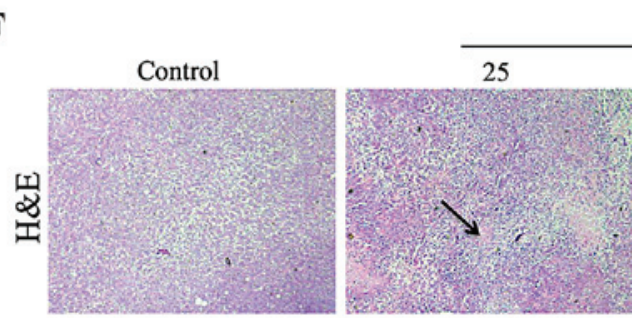
Canagliflozin (mg/kg)
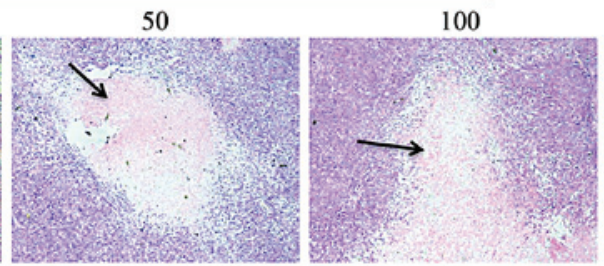

body weight

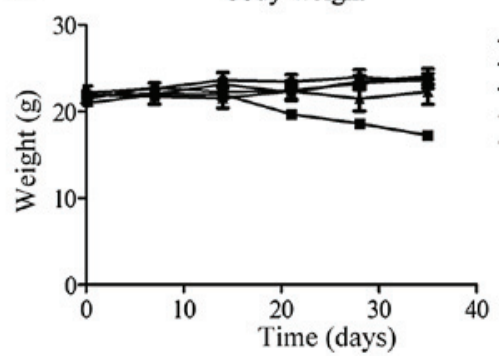

- Control $10 \mathrm{mg} / \mathrm{kg} \mathrm{GEM}$ $25 \mathrm{mg} / \mathrm{kg}$ CANA $50 \mathrm{mg} / \mathrm{kg}$ CANA $100 \mathrm{mg} / \mathrm{kg}$ CANA

Figure 4. CANA effectively inhibits PANC-1 tumor growth in nude mice. (A) Tumors harvested from the control and each treatment group were imaged. (B) The tumor inhibitory rate was increased in PANC-1 tumor-bearing nude mice treated with CANA. (C) The tumor weights and (D) tumor volumes were decreased in PANC-1-bearing mice treated with CANA in a dose-dependent manner. (E) The protective effect of CANA on body weight indicated that CANA did not affect the growth of mice. (F) Paraffin-embedded tumor sections were stained with $\mathrm{H} \& \mathrm{E}$. ${ }^{*} \mathrm{P}<0.05,{ }^{* * *} \mathrm{P}<0.01$ and ${ }^{* * * *} \mathrm{P}<0.001$. CANA, canagliflozin; $\mathrm{H} \& \mathrm{E}$, hematoxylin and eosin.

data. Thus, the data in the plots and graphs do not necessarily represent each other. The total apoptotic rate of the Capan-1 and PANC-1 cells increased from 20.0 and $19.8 \%$ to 34.3 and $35.5 \%$, respectively, when CANA $(60 \mu \mathrm{M})$ was used in combination with $25 \mu \mathrm{M}$ LY294002 (Fig. 3F and I). The data mentioned above suggest that LY294002 enhances the effect of CANA on the induction of the apoptosis of pancreatic cancer cells.

PANC-1 tumor growth is effectively inhibited during the treatment of nude mice with CANA. As CANA suppressed the growth of pancreatic cancer cells in vitro, the antitumor effects of this compound were further investigated in nude mice. The mice were subcutaneously implanted with PANC-1 cells $\left(100 \mu 1,6 \times 10^{6}\right.$ cells $\left./ \mathrm{ml}\right)$ and treated with CANA at 3 different doses, with gemcitabine alone or with the vehicle control. The group that received CANA exhibited an effective inhibition of tumor growth (Fig. 4A-D), with tumor inhibitory rates of 20.7, 29.4 and $45.1 \%$ at the doses of 25, 50 and $100 \mathrm{mg} / \mathrm{kg}$ in PANC-1 (Fig. 4B) nude mice, respectively. Similarly, the weights and volumes of the tumors were apparently decreased in a dose-dependent manner (Fig. 4C and D). Following 5 weeks of administration, the mean body weight of the CANA-treated groups was maintained at approximately $22 \mathrm{~g}$, whereas that of the mice in the GEM-treated group was significantly decreased to $17 \mathrm{~g}$ (Fig. 4E), suggesting that CANA was less toxic than the positive control drug, gemcitabine. Moreover, H\&E staining indicated the necrosis of parenchymal tumor tissue in the CANA-treated group compared with that of the control group (Fig. 4F). Collectively, these results indicated that CANA effectively inhibited the growth of PANC-1 tumors with minimal toxicity to the mice.

Glycolysis-associated $m R N A$ and protein expression levels are suppressed in vivo. To investigate whether the antitumor effects of CANA are associated with the inhibition of the glycolytic process, the mRNA and protein levels of glucose metabolism-associated genes were assayed by RT-qPCR and western blot analysis in the PANC-1-derived tumor tissues. The data indicated that the mRNA expression levels of GLUT-1 and LDHA were decreased by 71.5 and $56.6 \%$, respectively, at the dose of $25 \mathrm{mg} / \mathrm{kg}$ CANA (Fig. 5A and B). Concomitantly, the protein levels of PI3K, p-AKT, p-mTOR, HIF-1 $\alpha$, LDHA and GLUT-1 in the CANA-treated group were also decreased in the PANC-1-derived tumors (Fig. 5C and D), suggesting that glycolysis was inhibited by CANA via the PI3K/AKT/mTOR pathway in vivo. 
A

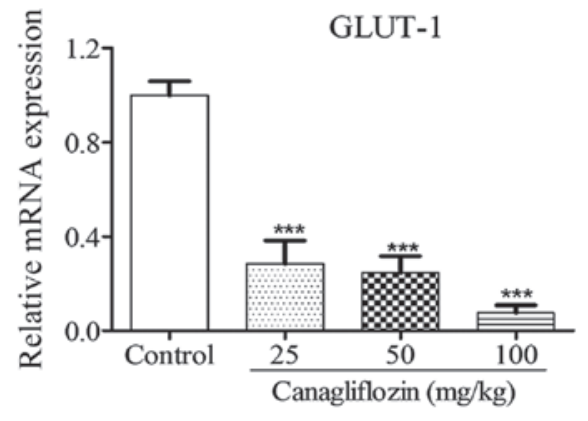

C

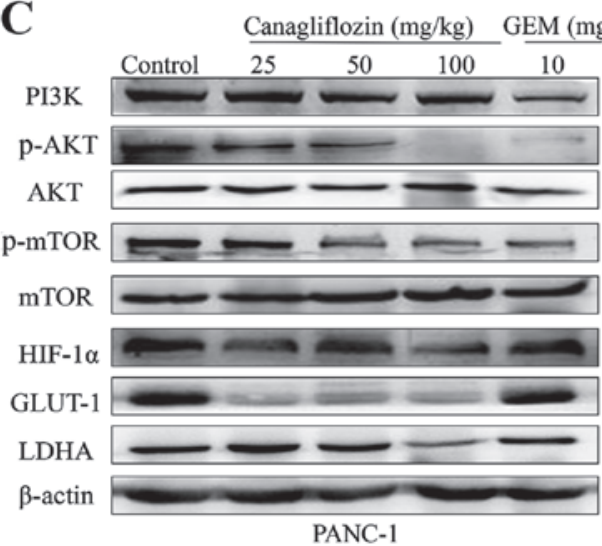

$\mathbf{B}$

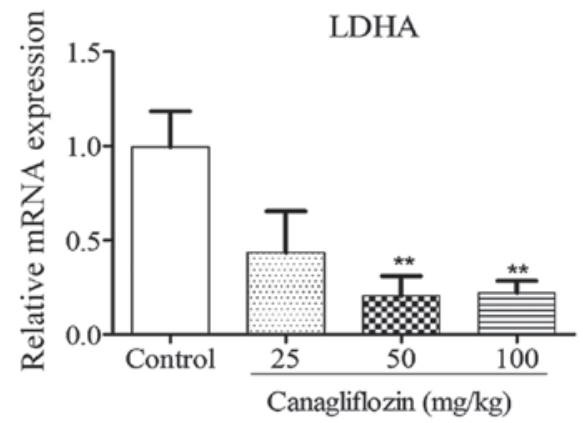

PANC-1

Control $\mathbf{0} 25 \mathrm{mg} / \mathrm{kg}$ 피 $100 \mathrm{mg} / \mathrm{kg}$

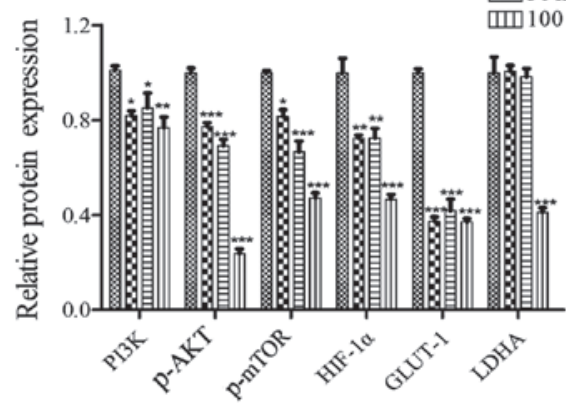

Figure 5. CANA dose-dependently suppresses the expression of GLUT-1 and LDHA, and PI3K/AKT/mTOR signaling in PANC-1 tumors. (A and B) Total RNA was extracted from PANC-1 tumors and the mRNA expression levels of GLUT-1 and LDHA were analyzed by RT-qPCR. (C) The protein levels of PI3K, p-AKT, p-mTOR, HIF-1 $\alpha$, GLUT-1 and LDHA were determined by western blot analysis and quantified by ImageJ software. (D) The relative expression levels were normalized to the control. Data are presented as the means \pm SD of 3 independent experiments, ${ }^{*} \mathrm{P}<0.05,{ }^{* *} \mathrm{P}<0.01$ and ${ }^{* * *} \mathrm{P}<0.001$ compared to the control. GLUT-1, glucose transporter-1; LDHA, lactate dehydrogenase A; CANA, canagliflozin; RT-qPCR, reverse transcription-quantitative PCR; p-, phosphorylated.

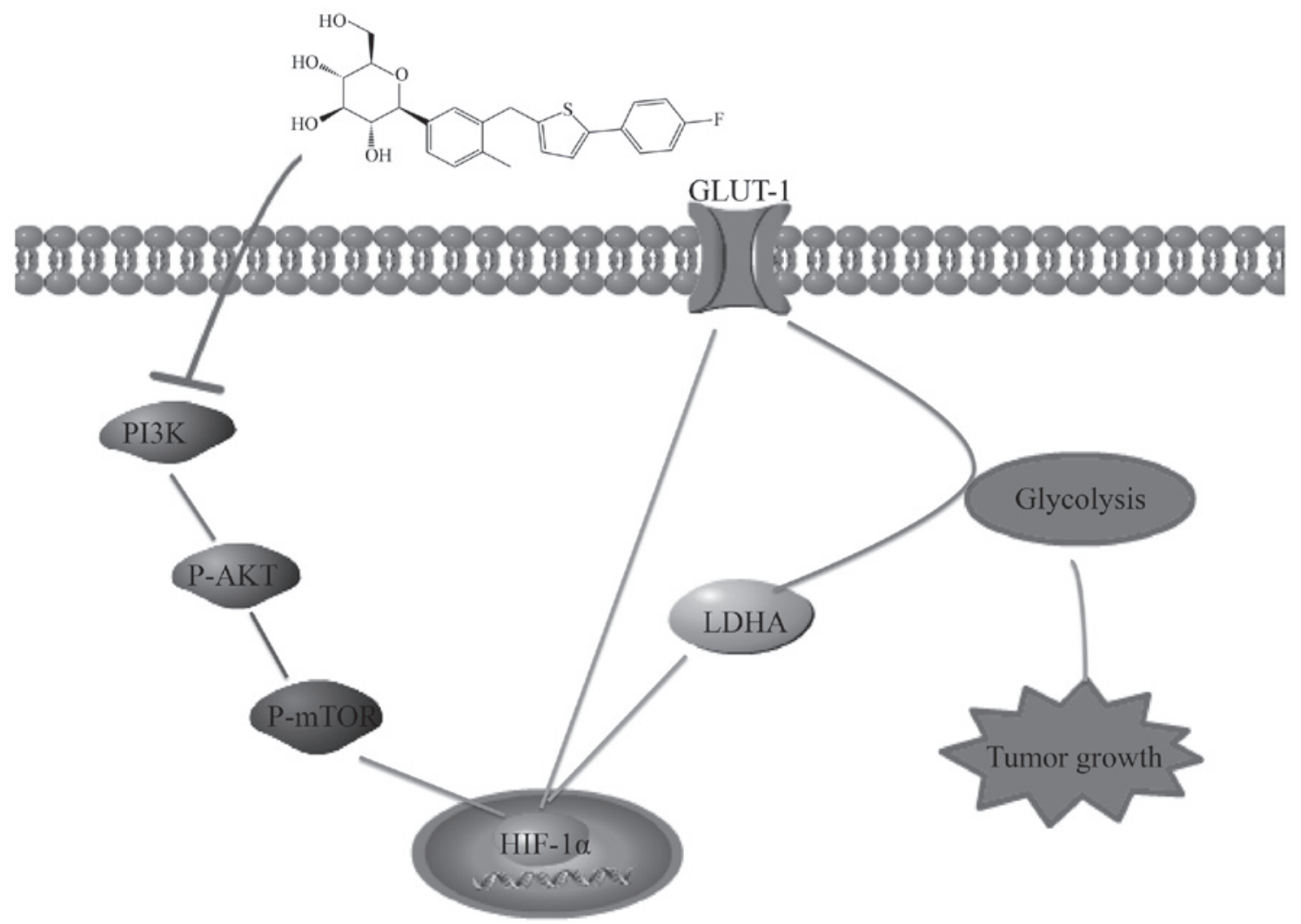

Figure 6. Proposed model of CANA function in treated pancreatic cancer cells. CANA inhibits PI3K/AKT/mTOR pathways to limit glycolysis and tumor growth. CANA, canagliflozin. 
CANA is capable of suppressing pancreatic cancer growth by inhibiting glycolysis. Cancer cells always reprogram their metabolism and enhance glycolysis to meet the constantly growing requirement for intermediate, the activation of the PI3K/AKT signaling pathway can promote pancreatic cancer proliferation. HIF-1 $\alpha$ is a major transcriptional regulator of the response to hypoxia that can induce several genes encoding glycolytic enzymes, such as glucose transporter-1 (GLUT-1), lactate dehydrogenase A (LDHA), which are highly expressed in tumors are involved in the glycolytic process (9). The ability of CANA to inhibit glycolysis is achieved by the downregulation of GLUT-1 and LDHA at the mRNA and protein levels via the PI3K/AKT/mTOR/HIF-1 $\alpha$ signaling pathway which effectively inhibiting the growth of pancreatic cancer (Fig. 6).

\section{Discussion}

As one of the most malignant and lethal solid cancers, pancreatic cancer is associated with insidious clinical symptoms, rapid progression, a poor prognosis and high mortality rates (20-22). This is probably due to low vascularization, prominent fibrotic stromal reactions, genomic complexity and metabolic alterations $(23,24)$. Currently, the first-line drug for the clinical treatment of pancreatic cancer causes significant side-effects, such as nephrotoxicity, allergic reactions and edema (25). Therefore, it is imperative to develop a safe and effective drug for treating pancreatic cancer. In the present study, the data indicated that CANA effectively inhibited the growth of pancreatic cancer cells (Fig. 1A and D) and suppressed the growth of PANC-1 tumors. The highest tumor inhibition rate reached $45.1 \%$ at doses of $100 \mathrm{mg} / \mathrm{kg}$ (Fig. 4A and B), suggesting that pancreatic cancer could be effectively inhibited during CANA treatment in vitro and/or in vivo. Moreover, the combination of CANA with gemcitabine improved the efficacy of gemcitabine in inhibiting pancreatic cancer cell proliferation and colony formation and inducing apoptosis in both cell lines (Figs. 1B, F and 2E). Furthermore, the apoptotic rate of the $40 \mu \mathrm{M}$ CANA-treated group was higher than that of the GEM-treated group, indicating that CANA could apparently induce pancreatic cancer cell apoptosis (Fig. 2A and D). The mean body weight of the CANA-treated mouse groups did not decrease, while that of the mice in the GEM-treated group was significantly reduced (Fig. 4E), suggesting that the toxicity of CANA was much lower than that of the positive drug gemcitabine. The data suggested that pancreatic cancer could be effectively inhibited by the low-toxicity drug CANA through the induction of apoptosis, which indicated its potential efficacy in clinical applications. However, further investigations are required to determine whether the cell cycle and metastatic activity of pancreatic cancer cells are affected by CANA. It was found by preliminary studies that the combined anti-tumor efficacy of CANA with gemcitabine was better than that of gemcitabine alone in Capan-1 and PANC-1 cells. In addition, the efficacy of the combined group in the mouse pancreatic cancer model and in the associated mechanisms can be studied to provide theoretical support for future clinical medication.

Otto Warburg was the first to demonstrate that tumor cells depend more on aerobic glycolysis accompanied by accelerated glucose uptake, which is currently known as the Warburg effect (6). The Warburg effect is a common trait of cancer, including pancreatic cancer $(19,26)$. Therefore, targeting metabolic reprogramming may be an extremely promising strategy for the treatment of pancreatic cancer. Everolimus is a clinically used drug for the treatment of advanced cancer. It inhibits human pancreatic cancer cell proliferation and induces apoptosis by interfering with glycolysis. The sodium glucose cotransporter 2 inhibitor, CANA, is currently used clinically in the treatment of diabetes. In the present study, the glucose uptake and lactate release rates of PANC-1 cells were reduced in the treated group ( $60 \mu \mathrm{M}$ CANA) by 4.9 and $43.3 \%$, respectively compared with those of the control group (Fig. 3A and B). This suggested that CANA inhibited pancreatic cancer progression by suppressing glucose metabolism, which is consistent with previous studies. It could be concluded that suppressing metabolic reprogramming is a potential option for pancreatic cancer treatment. However, only preliminary studies have been conducted on the suppression of glucose metabolism during treatment with CANA. To fully examine the mechanism by which CANA inhibits pancreatic cancer, it is necessary to further determine whether this compound affects the metabolic function of mitochondria.

Due to its polar hydrophilicity, glucose cannot freely pass through the cell membrane lipid bilayer structure and requires transportation via GLUT. Recent studies have reported that extracellular glucose can be transported into cancer cells via GLUT-1 in order to participate in the glycolytic process $(27,28)$. GLUT-1 and LDHA are overexpressed in pancreatic cancer $(29,30)$. The identification of a drug targeting GLUT-1 and LDHA may have significant applications in the treatment of pancreatic cancer. It has also been reported that HIF- $1 \alpha$ is associated with the PI3K/AKT/mTOR pathway (31) and that it induces the expression of genes involved in glycolysis $(32,33)$. Moreover, HIF-1 $\alpha$ stimulates the production of glycolytic energy by transactivating the gene GLUT-1 and by inducing LDHA to catalyze lactic acid conversion $(34,35)$. The results indicated that the mRNA expression levels of the glycolytic genes, including GLUT-1 and LDHA, were downregulated (Figs. 3C, 5A and B), whereas the protein levels of PI3K, p-AKT, p-mTOR, HIF-1 $\alpha$, GLUT-1 and LDHA were suppressed by CANA treatment to pancreatic cells. In order to determine whether CANA functions through the PI3K/AKT pathway, the combination of CANA with the PI3K/AKT pathway inhibitor, LY294002, on the apoptosis of PANC-1 and Capan-1 cells was investigated. Apparently, the total apoptotic rate of the Capan-1 and PANC-1 cells increased from 20.0 and $19.8 \%$ to 34.3 and $35.5 \%$, respectively (Fig. 3F and I), suggesting that LY294002 can enhance the effect of CANA on inducing apoptosis of pancreatic cancer cells. However, there has no enough evidence to clarify if it only functioned through the PI3K/AKT pathway or other pathways, which warrants further investigations. In contrast to these findings, gemcitabine did not induce these effects (Figs. 3D and 5C), suggesting that glycolysis in pancreatic cancer was reduced by the downregulation of GLUT-1 and LDHA expression caused through the PI3K/AKT/mTOR signaling pathway (Fig. 6). These effects were associated with the tumorigenic processes, including cell proliferation (Fig. 6). Currently, the additional molecules 
or pathways regulated by CANA have not been identified and warrant further investigation in future studies.

The exploration of new applications for existing drugs can reduce the cost and time required during drug development. The current findings provide novel evidence and demonstrate that CANA effectively inhibits the growth of pancreatic cancer in addition to its anti-diabetic role. CANA is capable of suppressing pancreatic cancer growth by inhibiting glycolysis, which is achieved by the downregulation of GLUT-1 and LDHA at the mRNA and protein levels via the $\mathrm{PI} 3 \mathrm{~K} / \mathrm{AKT} / \mathrm{mTOR} / \mathrm{HIF}-1 \alpha$ signaling pathway (Fig. 6). It is also interesting to note that the combination of CANA and gemcitabine exhibited an optimal therapeutic effect on pancreatic cancer cells, providing a theoretical basis and guidance for clinical drug use. These data provide important information for future targeted research on the use of CANA as a chemotherapeutic drug for other tumor types. It is expected that the addition of CANA as a novel chemotherapeutic drug may benefit pancreatic cancer patients treated with the first-line drug gemcitabine.

\section{Acknowledgements}

Not applicable.

\section{Funding}

The present study was supported by the MOST of China (2018YFA0902000, 2019ZX09721-001-04-01), the '111 Project' from the Ministry of Education of China (grant no. 111-2-07) and 'Double First-Class' University Project (grant no. CPU2018GF/GY16).

\section{Availability of data and materials}

The datasets used and/or analyzed during the current study are available from the corresponding author on reasonable request.

\section{Authors' contributions}

DX, YZ and CZ initiated the study, and conceived and performed the experiments. XX, LH and JD contributed to the data analysis. SP designed the combined administration protocol and provided the data. $\mathrm{BS}$ and $\mathrm{CZ}$ contributed to the conception of the study and assisted with data interpretation. All authors read and approved the final manuscript.

\section{Ethics approval and consent to participate}

All animal experiments were performed according to the protocols approved by the Ethics Committee of China Pharmaceutical University (approval no. SYXK 2016-0011).

\section{Patient consent for publication}

Not applicable.

\section{Competing interests}

The authors declare that they have no competing interests.

\section{References}

1. Siegel RL, Miller KD and Jemal A: Cancer statistics, 2016. CA Cancer J Clin 66: 7-30, 2016.

2. Garrido-Laguna I and Hidalgo M: Pancreatic cancer: From state-of-the-art treatments to promising novel therapies. Nat Rev Clin Oncol 12: 319-334, 2015.

3. Skandalakis LJ, Rowe JS Jr, Gray SW and Skandalakis JE: Surgical embryology and anatomy of the pancreas. Surg Clin North Am 73: 661-697, 1993.

4. Burris HA III, Moore MJ, Andersen J, Green MR, Rothenberg ML, Modiano MR, Cripps MC, Portenoy RK, Storniolo AM, Tarassoff $P$, et al: Improvements in survival and clinical benefit with gemcitabine as first-line therapy for patients with advanced pancreas cancer: A randomized trial. J Clin Oncol 15: 2403-2413, 1997.

5. Levine AJ and Puzio-Kuter AM: The control of the metabolic switch in cancers by oncogenes and tumor suppressor genes. Science 330: 1340-1344, 2010.

6. Warburg O: On the origin of cancer cells. Science 123: 309-314, 1956.

7. Vidal C, Rauly I, Zeggari M, Delesque N, Esteve JP, Saint-Laurent N, Vaysse N and Susini C: Up-regulation of somatostatin receptors by epidermal growth factor and gastrin in pancreatic cancer cells. Mol Pharmacol 46: 97-104, 1994.

8. Miller DM, Thomas SD, Islam A, Muench D and Sedoris K: c-Myc and cancer metabolism. Clin Cancer Res 18: 5546-5553, 2012.

9. Blum R and Kloog Y: Metabolism addiction in pancreatic cancer. Cell Death Dis 5: e1065, 2014.

10. Goldberg MS and Sharp PA: Pyruvate kinase M2-specific siRNA induces apoptosis and tumor regression. J Exp Med 209: 217-224, 2012.

11. Fantin VR, St-Pierre J and Leder P: Attenuation of LDH-A expression uncovers a link between glycolysis, mitochondrial physiology, and tumor maintenance. Cancer Cell 9: 425-434, 2006.

12. Kim YS and Milner JA: Bioactive food components and cancer-specific metabonomic profiles. J Biomed Biotechnol 2011: 721213, 2011.

13. Chao EC and Henry RR: SGLT2 inhibition-a novel strategy for diabetes treatment. Nat Rev Drug Discov 9: 551-559, 2010.

14. Kaji K, Nishimura N, Seki K, Sato S, Saikawa S, Nakanishi K, Furukawa M, Kawaratani H, Kitade M, Moriya K, et al: Sodium glucose cotransporter 2 inhibitor canagliflozin attenuates liver cancer cell growth and angiogenic activity by inhibiting glucose uptake. Int J Cancer 142: 1712-1722, 2018.

15. Shiba K, Tsuchiya K, Komiya C, Miyachi Y, Mori K, Shimazu N, Yamaguchi S, Ogasawara N, Katoh M, Itoh M, et al: Canagliflozin, an SGLT2 inhibitor, attenuates the development of hepatocellular carcinoma in a mouse model of human NASH. Sci Rep 8: 2362, 2018.

16. Livak KJ and Schmittgen TD: Analysis of relative gene expression data using real-time quantitative PCR and the 2(-Delta Delta C(T)) method. Methods 25: 402-408, 2001.

17. Jiang H, Fan D, Zhou G, Li X and Deng H: Phosphatidylinositol 3-kinase inhibitor(LY294002) induces apoptosis of human nasopharyngeal carcinoma in vitro and in vivo. J Exp Clin Cancer Res 29: 34, 2010.

18. Koppenol WH, Bounds PL and Dang CV: Otto Warburg's contributions to current concepts of cancer metabolism. Nat Rev Cancer 11: 325-337, 2011.

19. Gatenby RA and Gillies RJ: Why do cancers have high aerobic glycolysis? Nat Rev Cancer 4: 891-899, 2004.

20. Kamisawa T, Wood LD, Itoi T and Takaori K: Pancreatic cancer. Lancet 388: 73-85, 2016.

21. Chu LC, Goggins MG and Fishman EK: Diagnosis and detection of pancreatic Cancer. Cancer J 23: 333-342, 2017.

22. Dreyer SB, Chang DK, Bailey P and Biankin AV: Pancreatic cancer genomes: Implications for clinical management and therapeutic development. Clin Cancer Res 23: 1638-1646, 2017.

23. Le A, Rajeshkumar NV, Maitra A and Dang CV: Conceptual framework for cutting the pancreatic cancer fuel supply. Clin Cancer Res 18: 4285-4290, 2012.

24. Sousa CM and Kimmelman AC: The complex landscape of pancreatic cancer metabolism. Carcinogenesis 35: 1441-1450, 2014.

25. Heinrich S and Lang H: Neoadjuvant therapy of pancreatic Cancer: Definitions and benefits. Int J Mol Sci 18: 1622, 2017. 
26. Garber K: Energy deregulation: Licensing tumors to grow. Science 312: 1158-1159, 2006.

27. Yoon SO, Jeon TJ, Park JS, Ryu YH, Lee JH, Yoo JS, Kim JK, Yoon DS and Oh EJ: Analysis of the roles of glucose transporter 1 and hexokinase 2 in the metabolism of glucose by extrahepatic bile duct cancer cells. Clin Nucl Med 40: e178-e182, 2015.

28. Zhang TB, Zhao Y, Tong ZX and Guan YF: Inhibition of glucose-transporter 1 (GLUT-1) expression reversed Warburg effect in gastric cancer cell MKN45. Int J Clin Exp Med 8: 2423-2428, 2015.

29. Sun HC, Qiu ZJ, Liu J, Sun J, Jiang T, Huang KJ, Yao M and Huang C: Expression of hypoxia-inducible factor-1 alpha and associated proteins in pancreatic ductal adenocarcinoma and their impact on prognosis. Int J Oncol 30: 1359-1367, 2007.

30. Rong Y, Wu W, Ni X, Kuang T, Jin D, Wang D and Lou W: Lactate dehydrogenase $\mathrm{A}$ is overexpressed in pancreatic cancer and promotes the growth of pancreatic cancer cells. Tumor Biol 34: 1523-1530, 2013.
31. Li X, Wenes M, Romero P, Huang SC, Fendt SM and Ho PC: Navigating metabolic pathways to enhance antitumour immunity and immunotherapy. Nat Rev Clin Oncol 16: 425-441, 2019.

32. Wang V, Davis DA, Haque M, Huang LE and Yarchoan R: Differential gene up-regulation by hypoxia-inducible factor-1alpha and hypoxia-inducible factor-2alpha in HEK293T cells. Cancer Res 65: 3299-3306, 2005.

33. Gordan JD, Bertout JA, Hu CJ, Diehl JA and Simon MC: HIF-2alpha promotes hypoxic cell proliferation by enhancing c-myc transcriptional activity. Cancer Cell 11: 335-347, 2007.

34. Chen C, Pore N, Behrooz A, Ismail-Beigi F and Maity A: Regulation of glut 1 mRNA by hypoxia-inducible factor-1. Interaction between H-ras and hypoxia. J Biol Chem 276: 9519-9525, 2001.

35. Firth JD, Ebert BL and Ratcliffe PJ: Hypoxic regulation of lactate dehydrogenase A. Interaction between hypoxia-inducible factor 1 and cAMP response elements. J Biol Chem 270: 21021-21027, 1995. 